\title{
Predictive model of antimicrobial-resistant Gram-negative bacteremia at the ED
}

\author{
Wen-Chu Chiang MD, MPH ${ }^{a}$, Shey-Ying Chen MD ${ }^{a}$, Kuo-Liong Chien MD, PhD ${ }^{b}$, \\ Grace Hui-Min $\mathbf{W u}, \mathrm{PhD}^{\mathrm{b}}$, Amy Ming-Fang Yen $\mathrm{PhD}^{\mathbf{b}}$, Chan-Ping Su MDa, \\ Chien-Chang Lee MD $^{a}$, Yee-Chun Chen MD, PhDc, Shan-Chwen Chang MD, PhD $^{c}$, \\ Shyr-Chyr Chen MDa , Wen-Jone Chen MD, PhDa , Tony Hsiu-Hsi Chen DDS, PhD ${ }^{b}$,*
}

\author{
${ }^{a}$ Department of Emergency Medicine, National Taiwan University Hospital, Taipei 100, Taiwan \\ ${ }^{\mathrm{b}}$ Institute of Preventive Medicine, College of Public Health, National Taiwan University, Taipei 100, Taiwan \\ ${ }^{\mathrm{c}}$ Division of Infectious Disease, Department of Internal Medicine, National Taiwan University Hospital, Taipei 100, Taiwan
}

Received 17 August 2006; revised 30 November 2006; accepted 30 November 2006

\begin{abstract}
Background: Despite numerous studies identifying the risk factors related to Gram-negative antimicrobial resistance, an epidemiological model to reliably predict antimicrobial Gram-negative resistance in clinics, before the bacterial culture result is available, has not yet been developed.

Objectives: The aim of this study was to develop a predictive model to assist physicians in selecting appropriate antimicrobial agents before the details of the microbiology and drug susceptibility are known. Materials and Methods: A prospective study was conducted between June 1, 2001, and May 31, 2002, at the emergency department (ED) of National Taiwan University Hospital. Enrollees were patients with Gram-negative bacteremia (GNB) at ED. Other information collected included demographic characteristics, underlying comorbidities, hospital exposure and health care-associated factors, and details of initial presentation. Two primary outcomes were defined, including cefazolin-resistant (CZRES) GNB and ceftriaxone-resistant (CTX-RES) GNB. Two thirds of the data was randomly allocated to a derivation data set (for developing predictive models), and the rest, to a validation data set (for testing model validity). Simplified models, using a coefficient-based scoring method, were also developed for clinical applications.

Results: Based on 695 episodes of GNB, predictors of CZ-RES GNB were time since last hospitalization (increased risk for durations $<1$ month), prior infection with a CTX-RES strain, posttransplantation immunosuppressant use, residence in a nursing home or history of stroke with repeated choking, and poor oxygen saturation $(<95 \%)$ at admission to ED. Cirrhosis showed a protective effect by reducing the odds of antimicrobial-resistant GNB. The area under receiver operating characteristic (ROC) curve for the CZ-RES model was 0.76 (95\% confidence interval, 0.71-0.81).

The CTX-RES model included all the variables that were in the CZ-RES model plus abnormal
\end{abstract}

* Corresponding author. Tony Hsiu-Hsi Chen, Institute of Preventive Medicine, College of Public Health, National Taiwan University, Taipei 100, Taiwan. Tel.: +886 23516478 (25); fax: +8862 23920456. Address for reprints: Wen-Chu Chiang, Department of Emergency Medicine, National Taiwan University Hospital, Taipei 100, Taiwan. Tel.: +886 23562168; fax: +886223223150.

E-mail addresses: drchiang@ha.mc.ntu.edu.tw (W.-C. Chiang), stony@episerv.cph.ntu.edu.tw (T. Hsiu-Hsi Chen). 
leukocyte count $\left(<1000\right.$ or $\left.>15000 / \mathrm{mm}^{3}\right)$ at entry to ED. In this case, however, previous hospitalization within the last 2 weeks was a key factor. The area under this ROC curve was 0.82 (95\% confidence interval, 0.76-0.88). There was lacking of difference in the area under the ROC curve between the 2 final (simplified) models either based on the derivation or validation data sets.

Conclusion: We have developed 2 models for predicting risk of antimicrobial Gram-negative infection by identifying and quantifying associated risk factors. These models could be used by physicians to determine the most appropriate choice of antibiotic for first-line therapy, particularly in situations where the culture result is not yet known.

(C) 2007 Elsevier Inc. All rights reserved.

\section{Introduction}

Gram-negative bacterial infections are becoming increasingly prevalent in many populations and have, because of their association with unfavorable clinical outcomes, attracted much attention over the past decade. In particular, such infections often lead to longer hospital stays, increased attributable mortality, and greater hospitalization costs [1-5]. The identification of risk factors affecting susceptibility to antibiotic resistant Gram-negative pathogens is therefore of paramount importance.

Risk factors identified in previous studies included demographic characteristics, underlying comorbidities, and the type or pattern of medical care. Subjects who are older than 65 years have been reported as being a high-risk group [6], although few studies have corroborated this. Having hepatic failure or end-stage renal disease [7], or having had gastrointestinal (GI) surgery or an organ transplant [8-10] makes infection with an antimicrobialresistant Gram-negative pathogen more likely. Although diabetes mellitus is more susceptible to infection, it was not associated with antimicrobial resistance in many reviews [6-16]. Hospital exposure and health care-associated factors were investigated in many studies, more specifically, long-term hemodialysis at clinics or hospital $[11,12]$, residence in a nursing-home $[12,13]$, history of prolonged hospital stays $[8,9,14]$ having an indwelling catheter (such as central venous, arterial $[7,13]$, or urinary) $[7,15,16]$, requiring mechanical ventilation $[7,15,16]$, and being tube-fed [10]. Previous anti-infective treatment with fluroquinolones $[8,9]$ and multiple antibiotic exposure lasting longer than 14 days $[6,10]$ were also considered as risk factors.

There are 3 deficiencies in these previous findings. Firstly, most of the research was based on retrospective studies that used a slightly different definition of antibiotic resistance, making the results difficult to interpret. Secondly, with so many risk factors identified, a physician may be faced with an impossible task when trying to choose an appropriate antibiotic. Finally, as antimicrobial resistance develops through the use of antibiotics, which varies from country to country, the findings from studies carried out in the west may not be generalized to other populations.
As cases of antimicrobial-resistant Gram-negative bacterial infection differ considerably as regards host factors, the objective of this study was to use background patient information to develop a series of predictive models that could help physicians identify high-risk patients and decide which antimicrobial agent to prescribe.

\section{Materials and methods}

\subsection{Design, setting, and enrollees}

Patients with bacteremia were identified prospectively during the period June 1, 2001, through May 31, 2002, from attendees at the emergency department (ED) of National Taiwan University Hospital (NTUH) in Taipei, which has a capacity of 2400 beds and more than 100000 ED visits annually. Note that only nontrauma adult patients (ie, $>15$ years old) with proven Gram-negative bacteremia (GNB) were enrolled in this study. The details of study process from initial report of bacterial growth to the identification of exposure and outcome assessment are summarized in Fig. 1. This study was approved by the institutional review board of NTUH.

Cephalosporins were extensively used to treat Gramnegative bacterial infections in Taiwan [17]. First-generation cephalosporin remains sensitive to most Gram-positive and Gram-negative pathogens. When the in vitro test shows susceptibility, they are a better choice because they are inexpensive and induce resistance less than the second- and third-generation cephalosporins $[18,19]$. In contrast, thirdgeneration cephalosporin has the widest spectrum of Gramnegative activity and is preferred in most serious Gramnegative infection. Furthermore, if the pathogen is suspected of being nonfermenting, Gram-negative bacilli or extendedspectrum $\beta$-lactamase Enterobacter spp, a stronger antimicrobial agent, such as imipenem, will be prescribed.

\subsection{Exposures assessment}

To develop a predictive model for antimicrobial-resistant bacteremia, we collected information on as many of the previously identified predictors of antimicrobial-resistant Gram-negative bacterial infection as possible. Table 1 specifies 60 items of exposures, including 4 blanket terms: 


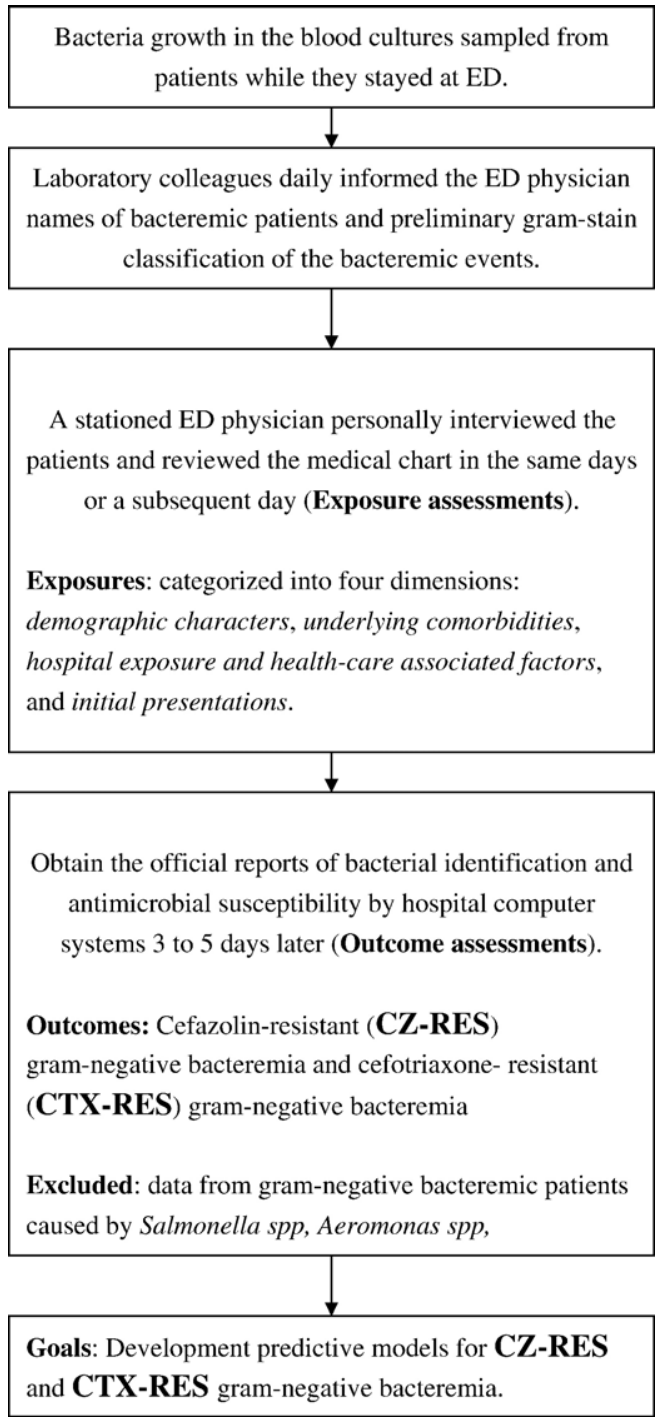

Fig. 1 Flowchart of study design.

demographic characteristics, underlying comorbidities, hospital exposure and health care-associated factors, and initial presentation. Medical history and clinical presentations of all eligible patients were obtained by person-toperson interview and detail medical chart review by the stationed physician. During this evaluation stage, the physician who carried out all the questionnaire interviews and chart reviews was unaware of the details of the infection and blind to the outcome of antibiotic resistance because the final microbiology report would not have been available until 3 to 5 days after the first laboratory telephone report.

\subsection{Outcome management}

We set out 2 prediction models to assist physicians' in choosing antibiotics. The first model is related to the resistance to first-generation cephalosporin, defined as cefazolin-resistant (CZ-RES) GNB, and the second is pertinent to the resistance to third-generation cephalosporin (without anti-Pseudomonas activity), defined as ceftriaxone-resistant (CTX-RES) GNB. Data relating to bacteremic episodes caused by Salmonella spp, Aeromonas spp, Vibrio spp, or Campylobacter spp were excluded from analysis because the clinical features, predominant risk factors, and prescribing practice with such infections were largely different from those of the most common Gram-negative infections [20-25].

\subsection{Microbiological assessments and antimicrobial susceptibility}

Blood from patients was stored in BACTEC standard or BACTEC PLUS culture bottles in a BACTEC 9000 system (Becton Dikinson, Cockeysville, Md) for blood culture. Identification of the isolates and susceptibility testing was performed at the central laboratory in NTUH using standard bacteriologic techniques and an automated system (VITEK, bioMerieux Inc, USA).

\subsection{Statistical method for predictive models}

We adapted derivation-validation method to develop predictive models. Detailed processes of the enrollment and randomization are given in Fig. 2. Continuous data were categorized by splitting into groups with appropriately chosen cut points. Univariate associations between categor-

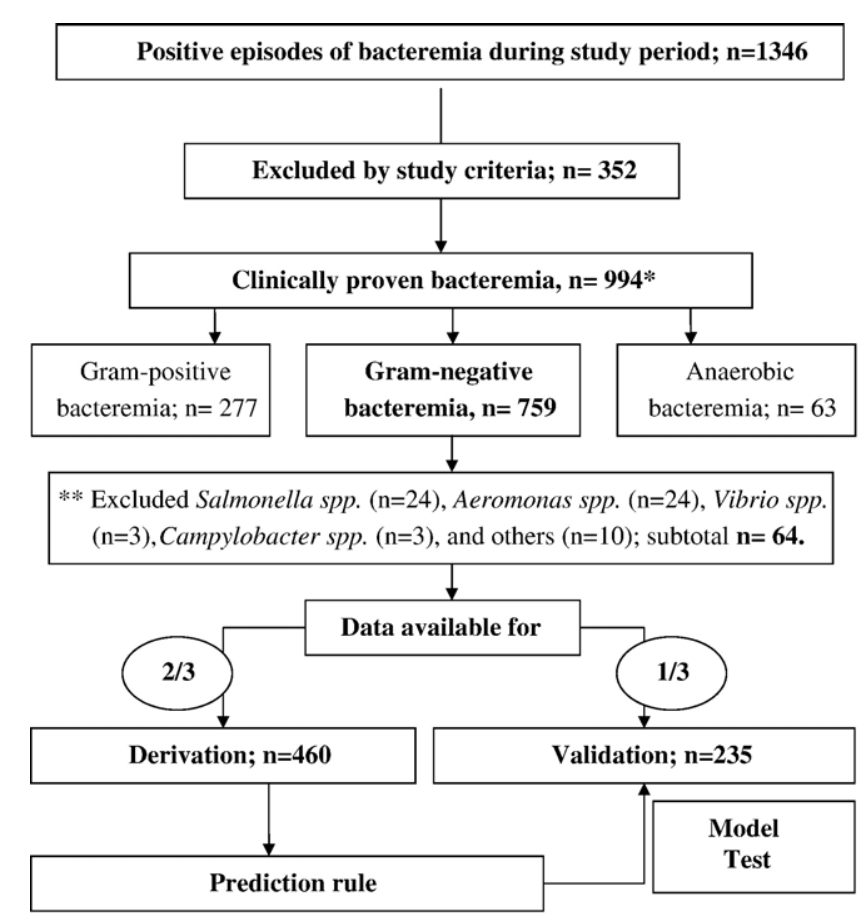

* The number of episodes of clinically proven bacteremia was less than the sum of the numbers with gram-positive, gram-negative and anaerobic bacteremia because some episodes were poly-microbial bacteremia.

Fig. 2 Enrollment, data collection, and assignment. 
Table 1 Univariate analyses of derivation data set $(n=460)$

\begin{tabular}{|c|c|c|c|c|c|c|c|}
\hline \multirow[t]{2}{*}{ Exposure: E (+) } & \multirow[t]{2}{*}{ Total E (+) } & \multicolumn{3}{|c|}{ CZ-RES } & \multicolumn{3}{|c|}{ CTX-RES } \\
\hline & & Cases & OR & $95 \% \mathrm{CI}$ & Cases & OR & $95 \% \mathrm{CI}$ \\
\hline \multicolumn{8}{|l|}{ Demographic factors } \\
\hline \multicolumn{8}{|l|}{ Sex } \\
\hline Male & 230 & 74 & $1.5^{*}$ & $1.0-2.3$ & 35 & 1.3 & $0.8-2.2$ \\
\hline female & 230 & 55 & 1.0 & - & 28 & 1.0 & - \\
\hline \multicolumn{8}{|l|}{ Age (y) } \\
\hline$\leq 40$ & 53 & 15 & 1.0 & - & 7 & 1.0 & - \\
\hline $41-64$ & 145 & 35 & 0.8 & $0.4-1.6$ & 221.2 & $0.5-2.9$ & \\
\hline $65-75$ & 128 & 37 & 1.0 & $0.5-2.1$ & 14 & 0.8 & $0.3-2.1$ \\
\hline$\geq 75$ & 134 & 42 & 1.2 & $0.6-2.3$ & 20 & 1.2 & $0.5-2.9$ \\
\hline \multicolumn{8}{|l|}{ Comorbidity factors } \\
\hline Diabetes mellitus & 139 & 38 & 1.0 & $0.6-1.5$ & 18 & 0.9 & $0.5-1.6$ \\
\hline Liver cirrhosis & 48 & 6 & $0.3^{*}$ & $0.1-0.8$ & 2 & 0.3 & $0.1-1.1$ \\
\hline End-stage renal disease & 11 & 4 & 1.5 & $0.4-5.2$ & 2 & 1.4 & $0.3-6.7$ \\
\hline Stroke with repeat choking history & 52 & 27 & 3.2.** & $1.8-5.8$ & 16 & $3.4 * *$ & $1.8-6.6$ \\
\hline \multicolumn{8}{|l|}{ Bedridden } \\
\hline Bedridden, no bedsore & 46 & 22 & $2.7 * *$ & $1.5-5.1$ & 13 & $3.0 * *$ & $1.5-6.2$ \\
\hline Bedridden, with bedsore & 13 & 6 & 2.6 & $0.8-7.8$ & 4 & $3.4^{*}$ & $1.0-11.6$ \\
\hline Alcohol consumption & 7 & 1 & 0.4 & $0.1-3.6$ & 0 & - & - \\
\hline Long-term steroid needed & 14 & 4 & 1.0 & $0.3-3.3$ & 2 & 1.1 & $0.2-4.8$ \\
\hline Hematological malignancy & 25 & 15 & $4.2 * *$ & $1.9-9.7$ & 8 & $3.3 * *$ & $1.3-7.8$ \\
\hline \multicolumn{8}{|l|}{ Solid organ malignancy } \\
\hline GI tract malignancy & 62 & 19 & 1.7 & $0.8-3.9$ & 11 & $4.1 * *$ & $1.7-9.7$ \\
\hline Non-GI tract malignancy & 26 & 10 & 1.2 & $0.7-2.2$ & 9 & 1.7 & $0.8-3.4$ \\
\hline Chemotherapy & 36 & 18 & $2.8 * *$ & $1.4-5.6$ & 13 & $2.8 * *$ & $1.4-5.6$ \\
\hline Immunosuppressant & 10 & 4 & 1.7 & $0.5-6.3$ & 2 & 1.6 & $0.3-7.9$ \\
\hline Autoimmune disease & 9 & 3 & 1.3 & $0.3-5.2$ & 2 & 1.8 & $0.4-9.0$ \\
\hline \multicolumn{8}{|l|}{ Health care-associated factors } \\
\hline \multicolumn{8}{|l|}{ Patient source } \\
\hline Community & 390 & 91 & 1.0 & - & 42 & 1.0 & - \\
\hline Nursing home & 16 & 10 & $5.5^{* *}$ & $1.9-15.5$ & 5 & $3.8^{*}$ & $1.3-11.4$ \\
\hline Long-term health care needed & 30 & 11 & 1.9 & $0.9-4.2$ & 4 & 1.3 & $0.4-3.8$ \\
\hline Hospitalization in last month & 24 & 17 & $8.0 * *$ & $3.2-19.8$ & 12 & $8.3 * *$ & $3.5-19.6$ \\
\hline \multicolumn{8}{|l|}{ Time since the last discharge (d) } \\
\hline$<7$ & 46 & 30 & $8.3 * *$ & $4.2-16.5$ & 21 & $10.9 * *$ & $5.1-23.4$ \\
\hline $8-14$ & 19 & 9 & $4.0 * *$ & $1.5-10.4$ & 6 & $6.0 * *$ & $2.0-17.8$ \\
\hline $15-21$ & 20 & 11 & $5.4^{* *}$ & $2.1-13.8$ & 1 & 0.7 & $0.1-5.4$ \\
\hline $22-30$ & 19 & 8 & $3.2 *$ & $1.2-8.4$ & 4 & $3.5^{*}$ & $1.1-11.6$ \\
\hline $31-45$ & 18 & 4 & 1.3 & $0.4-4.0$ & 4 & $3.7 *$ & $1.1-12.5$ \\
\hline $46-365$ & 100 & 23 & 1.3 & $0.8-2.3$ & 10 & 1.4 & $0.6-3.3$ \\
\hline No hospitalization with the last year & 238 & 44 & 1.0 & - & 17 & 1.0 & - \\
\hline \multicolumn{8}{|l|}{ Last admitted hospital } \\
\hline No hospitalization with the last year & 238 & 44 & 1.0 & - & 17 & 1.0 & - \\
\hline Admitted, not at NTUH & 37 & 21 & $5.8^{* *}$ & $2.8-12.0$ & 12 & $6.2 * *$ & $2.7-14.6$ \\
\hline Admitted at NTUH & 165 & 64 & $2.3 * *$ & $1.5-3.6$ & 34 & $2.9^{* *}$ & $1.6-5.4$ \\
\hline \multicolumn{8}{|l|}{ Length of last hospital stay (d) } \\
\hline No hospitalization within last year & 238 & 46 & 1.0 & - & 17 & 1.0 & - \\
\hline$<7$ & 54 & 22 & $14.1 * *$ & $4.9-40.6$ & 12 & $14.3 * *$ & $5.3-38.4$ \\
\hline $8-15$ & 65 & 22 & $3.4 * *$ & $2.1-5.5$ & 11 & $3.8^{* *}$ & $2.0-7.3$ \\
\hline $16-30$ & 58 & 23 & 1.1 & $0.5-2.3$ & 13 & 1.1 & $0.4-3.4$ \\
\hline$>30$ & 43 & 16 & 1.3 & $0.5-3.3$ & 10 & 2.0 & $0.6-6.4$ \\
\hline \multicolumn{8}{|l|}{ Cause of prior hospitalization } \\
\hline No hospitalization with the last year & 238 & 44 & 1.0 & - & 17 & 1.0 & - \\
\hline Infectious disease & 144 & 60 & $2.7 *$ & $1.2-5.8$ & 33 & $2.1 *$ & $1.2-3.8$ \\
\hline Noninfectious disease & 77 & 25 & $3.9 * *$ & $2.1-7.3$ & 13 & $3.2 * *$ & $2.0-5.0$ \\
\hline
\end{tabular}


Table 1 (continued)

\begin{tabular}{|c|c|c|c|c|c|c|c|}
\hline \multirow[t]{2}{*}{ Exposure: E (+) } & \multirow[t]{2}{*}{ Total E (+) } & \multicolumn{3}{|c|}{ CZ-RES } & \multicolumn{3}{|c|}{ CTX-RES } \\
\hline & & Cases & OR & $95 \% \mathrm{CI}$ & Cases & OR & $95 \% \mathrm{CI}$ \\
\hline \multicolumn{8}{|l|}{ Prior infectious sites } \\
\hline None & 316 & 69 & 1.0 & - & 30 & 1.0 & - \\
\hline Low respiratory tract & 20 & 8 & 2.4 & $0.9-6.1$ & 6 & $4.1 * *$ & $1.5-11.4$ \\
\hline Intra-abdominal & 45 & 12 & 1.3 & $0.6-2.7$ & 7 & 1.8 & $0.7-4.3$ \\
\hline Urinary tract & 34 & 12 & 2.0 & $0.9-4.1$ & 8 & $2.9^{*}$ & $1.2-7.1$ \\
\hline Others & 26 & 16 & $5.7 * *$ & $2.5-13.2$ & 9 & $5.1 * *$ & $2.1-12.3$ \\
\hline Prior antibiotic exposure & 166 & 69 & $2.8 * *$ & $1.8-4.2$ & 37 & $3.0 * *$ & $1.7-5.1$ \\
\hline \multicolumn{8}{|l|}{ Prior infection with resistant strain } \\
\hline CZ-RES & 25 & 12 & $2.5^{*}$ & $1.1-5.7$ & 7 & $2.6^{*}$ & $1.1-6.6$ \\
\hline CTX-RES & 56 & 28 & $3.0 * *$ & $1.7-5.3$ & 17 & $3.4 * *$ & $1.8-6.5$ \\
\hline Vancomycin needed at OPD & 20 & 13 & $5.2 * *$ & $2.0-13.3$ & 8 & $4.7 * *$ & $1.8-11.9$ \\
\hline \multicolumn{8}{|l|}{ Procedure at OPD } \\
\hline No procedure needed at OPD & 423 & 115 & 1.0 & - & 56 & 1.0 & - \\
\hline Chemotherapy at OPD & 19 & 3 & 0.5 & $0.1-1.8$ & 1 & 0.4 & $0.1-2.8$ \\
\hline Hemodialysis & 7 & 4 & 1.9 & $0.9-4.0$ & 2 & 1.6 & $0.7-3.7$ \\
\hline Others & 11 & 7 & 0.6 & $0.1-5.8$ & 4 & $1.6^{*}$ & $1.0-2.4$ \\
\hline Mechanical valve & 5 & 1 & 0.6 & $0.1-5.7$ & 0 & - & - \\
\hline Arteriovenous fistula for dialysis & 9 & 4 & 2.1 & $0.6-8.0$ & 2 & 1.8 & $0.4-9.0$ \\
\hline Intravascular catheter & 7 & 5 & $3.1 * *$ & $1.7-5.6$ & 3 & $3.0 * *$ & $1.5-5.9$ \\
\hline Foley catheter & 16 & 10 & $4.6^{* *}$ & $1.6-12.8$ & 8 & $7.1 * *$ & $2.6-19.6$ \\
\hline Other urinary drainage & 5 & 3 & 3.9 & $0.7-23.7$ & 0 & - & - \\
\hline Missed discharged at ED* & 34 & 10 & 1.1 & $0.5-2.3$ & 5 & 1.1 & $0.4-2.9$ \\
\hline Recalling to ED & 20 & 8 & 1.8 & $0.7-4.4$ & 4 & 1.6 & $0.5-5.0$ \\
\hline \multicolumn{8}{|l|}{ Initial presentations } \\
\hline \multicolumn{8}{|l|}{ Fever duration } \\
\hline No fever & 124 & 36 & 1.0 & - & 20 & 1.0 & - \\
\hline $0-3 \mathrm{~d}$ & 294 & 77 & 0.9 & $0.5-1.4$ & 35 & 0.7 & $0.4-1.3$ \\
\hline$>4 \mathrm{~d}$ & 42 & 16 & 1.5 & $0.7-3.1$ & 8 & 1.2 & $0.5-3.0$ \\
\hline \multicolumn{8}{|l|}{ Systolic blood pressure $(\mathrm{mm} \mathrm{Hg})$} \\
\hline Low $(\leq 90)$ & 96 & 39 & $1.9^{*}$ & $1.2-3.2$ & 21 & 1.8 & $1.0-3.4$ \\
\hline Normal (91-140) & 209 & 55 & 1.0 & - & 28 & 1.0 & - \\
\hline High $(\geq 141)$ & 155 & 35 & 0.8 & $0.5-1.3$ & 14 & 0.6 & $0.3-1.3$ \\
\hline \multicolumn{8}{|l|}{ Diastolic blood pressure $(\mathrm{mm} \mathrm{Hg})$} \\
\hline Low $(\leq 60)$ & 72 & 38 & $3.4 * *$ & $2.0-5.7$ & 20 & $2.9 * *$ & $1.6-5.3$ \\
\hline Normal (60-89) & 330 & 82 & 1.0 & - & 39 & 1.0 & - \\
\hline High $(\geq 90)$ & 58 & 9 & $0.6^{*}$ & $0.3-1.2$ & 4 & 0.6 & $0.2-1.6$ \\
\hline \multicolumn{8}{|l|}{ Body temperature $\left({ }^{\circ} \mathrm{C}\right)$} \\
\hline$<35.9$ & 31 & 14 & $2.2 *$ & $1.0-4.7$ & 9 & $2.6^{*}$ & $1.1-6.1$ \\
\hline $36-38.2$ & 210 & 58 & 1.0 & - & 29 & 1.0 & - \\
\hline $38.3-39.4$ & 154 & 43 & 1.0 & $0.6-1.6$ & 20 & 0.9 & $0.5-1.7$ \\
\hline$>39.5$ & 65 & 14 & 0.7 & $0.4-1.4$ & 5 & 0.5 & $0.2-1.4$ \\
\hline \multicolumn{8}{|l|}{ Heart rate (per min) } \\
\hline$\leq 59$ & 5 & 1 & 0.6 & $0.1-5.8$ & 1 & 1.8 & $0.2-17.2$ \\
\hline $60-99$ & 140 & 40 & 1.0 & - & 17 & 1.0 & - \\
\hline$\geq 100$ & 315 & 88 & 1.0 & $0.6-1.5$ & 45 & 1.0 & $0.7-2.2$ \\
\hline \multicolumn{8}{|l|}{ Oxygen saturation } \\
\hline $\mathrm{SpO}_{2}=96-100 \%$ & 426 & 118 & 1.0 & - & 57 & 1.0 & - \\
\hline $\mathrm{SpO}_{2}<95 \%$ & 34 & 11 & 1.5 & $1.0-2.3$ & 6 & $2.4 * *$ & $1.4-4.1$ \\
\hline \multicolumn{8}{|l|}{ WBC count $\left(10^{9} / \mathrm{L}\right)$} \\
\hline$\leq 1000$ & 20 & 11 & $4.0 * *$ & $1.6-10.1$ & 8 & $6.7 * *$ & $2.5-17.9$ \\
\hline $1001-5000$ & 47 & 13 & 1.3 & $0.6-2.5$ & 10 & $2.7 * *$ & $1.2-6.1$ \\
\hline $5001-15000$ & 287 & 67 & 1.0 & - & 26 & 1.0 & - \\
\hline$\geq 15001$ & 106 & 38 & $1.8^{*}$ & $1.1-3.0$ & 19 & $2.2 *$ & $1.2-4.2$ \\
\hline Neutrophil percentage of WBC & & & & & & & \\
\hline
\end{tabular}


Table 1 (continued)

\begin{tabular}{|c|c|c|c|c|c|c|c|}
\hline \multirow[t]{2}{*}{ Exposure: E $(+)$} & \multirow[t]{2}{*}{ Total E (+) } & \multicolumn{3}{|c|}{ CZ-RES } & \multicolumn{3}{|c|}{ CTX-RES } \\
\hline & & Cases & OR & $95 \% \mathrm{CI}$ & Cases & OR & $95 \% \mathrm{CI}$ \\
\hline$\leq 49$ & 25 & 17 & $6.3^{* *}$ & $2.3-16.9$ & 12 & $6.4 * *$ & $2.2-18.2$ \\
\hline $50-80$ & 71 & 18 & 1.0 & - & 9 & 1.0 & - \\
\hline$\geq 81$ & 355 & 90 & 1.0 & $0.6-2.0$ & 40 & 0.9 & $0.4-1.9$ \\
\hline \multicolumn{8}{|c|}{ Band-from percentage of WBC } \\
\hline No band-form cell & 337 & 94 & 1.0 & - & 48 & 1.0 & - \\
\hline $119 \%$ & 31 & 7 & 0.8 & $0.3-1.8$ & 1 & 0.1 & $0.0-1.5$ \\
\hline$\geq 10 \%$ & 83 & 23 & 1.0 & $0.6-1.7$ & 12 & 1.0 & $0.5-2.0$ \\
\hline \multicolumn{8}{|c|}{ Hemoglobin level (g/dL) } \\
\hline$<10$ & 146 & 51 & $1.6^{*}$ & $1.0-2.5$ & 32 & $2.6^{* *}$ & $1.5-4.5$ \\
\hline $10.1-12.9$ & 272 & 68 & 1.0 & - & 27 & 1.0 & - \\
\hline$>13$ & 42 & 10 & 0.9 & $0.4-2.0$ & 4 & 1.0 & $0.3-2.9$ \\
\hline \multicolumn{8}{|c|}{ Thrombocyte count $\left(10^{9} / \mathrm{L}\right)$} \\
\hline$\leq 99$ & 123 & 40 & 1.4 & $0.9-2.2$ & 22 & 1.6 & $0.9-2.9$ \\
\hline $100-399$ & 320 & 82 & 1.0 & - & 38 & 1.0 & - \\
\hline$\geq 400$ & 17 & 7 & 2.0 & $0.8-5.5$ & 3 & 1.6 & $0.4-5.8$ \\
\hline
\end{tabular}

ical variables and the outcomes (CZ-RES and CTX-RES) were then assessed using the $\chi^{2}$ test or Fisher exact test, as appropriate. If a test was significant $(P<.05)$, the corresponding variable was considered to be univariately predictive of outcome. All variables showing an association with outcome $(P<.25)$ were entered into the multivariate logistic regression analysis.

We used stepwise method to select the parsimonious model. Collinearity of covariates was assessed by the variance inflation factor. Receiver operating characteristic (ROC) curves were constructed for assessing predictive validity on the basis of the area under the curve (AUC) and their confidence intervals (CIs) calculated with HanleyMcNeil formula [26]. The model fitting was assessed using the Hosmer-Lemeshow goodness-of-fit test.

To make the prediction models easy to use, we simplified them to make a prediction rule [27,28] using a regression coefficient-based scoring method. With this, a simple integer-based point score was generated for each predictor by doubling the value of the corresponding $\beta$-coefficient in the model and rounding up to the nearest integer. The overall risk score was calculated by adding the individual scores for each of the predictors together [29]. The resulting total scores were grouped to demonstrate the predictive performance of the models. All analyses were performed using SAS software version 9.1 (SAS Institute Inc, Cary, NC).

\section{Results}

There was a total of 1346 episodes of positive bacteremia identified at the ED during the study period, of which 695 episodes were of Gram-negative infection from 659 patients, with 25 patients having repeated episodes within a year. As shown in Fig. 2, these 695 episodes were randomly allocated (in the ratio of $2: 1)$ to the derivation and validation groups. Univariate analyses of collected variables were listed in Table 1. Of the demographic characteristics, there were 349 men and 310 women; average age of study population was $63.9 \pm$

Table 2 Predictors and assigned scores from the multivariate analysis of CZ-RES model

\begin{tabular}{|c|c|c|c|c|c|}
\hline Parameter & $\beta$-Coefficient & OR & $95 \% \mathrm{CI}$ & $P$ & Scores \\
\hline Time since last discharge: within $7 \mathrm{~d}$ & 2.1492 & 8.6 & $4.2-17.4$ & $<.0001$ & 4 \\
\hline Time since last discharge: between 8 and $30 \mathrm{~d}^{\mathrm{a}}$ & 1.5444 & 4.7 & $2.4-9.0$ & $<.0001$ & 3 \\
\hline Immunosuppressant use & 1.3610 & 3.9 & $1.0-15.1$ & .0488 & 3 \\
\hline Prior infection with a CTX-RES strain & 0.9694 & 2.6 & $1.4-5.0$ & .0039 & 3 \\
\hline Residence in a nursing home or stroke with history of repeated choking & 1.1062 & 3.0 & $1.6-5.7$ & .0006 & 2 \\
\hline Oxygen saturation $<95 \%{ }^{\mathrm{a}}$ & 0.6009 & 1.8 & $1.1-3.0$ & .0209 & 1 \\
\hline Liver cirrhosis & -1.2143 & 0.2 & $0.1-0.6$ & .0043 & -2 \\
\hline
\end{tabular}

${ }^{\text {a }}$ Nonpredictors or different assigned scores in comparing to CTX-RES model. 
Table 3 Predictors and assigned scores for the CTX-RES model

\begin{tabular}{|c|c|c|c|c|c|}
\hline Parameter & $\beta$-Coefficient & OR & $95 \% \mathrm{CI}$ & $P$ & Scores \\
\hline Time since last discharge: within $7 \mathrm{~d}$ & 2.2256 & 9.3 & $4.3-20.1$ & $<.0001$ & 4 \\
\hline Time since last discharge: between 8 and $14 \mathrm{~d}^{*}$ & 1.1744 & 3.2 & $0.9-11.2$ & .063 & 2 \\
\hline Prior infection with a CTX-RES strain & 1.2874 & 3.6 & $1.6-8.1$ & .0017 & 3 \\
\hline Immunosuppressant use & 1.6440 & 5.2 & $0.9-28.8$ & .0606 & 3 \\
\hline WBC count $>1000 / \mathrm{mm}^{3} *$ & 1.3278 & 3.9 & $1.8-8.8$ & .0008 & 3 \\
\hline Oxygen saturation $<95 \% *$ & 1.2155 & 3.4 & $1.7-6.6$ & .0004 & $2 *$ \\
\hline Residence in a nursing home or stroke with history of repeated choking & 1.0725 & 2.9 & $1.3-6.4$ & .0078 & 2 \\
\hline WBC count $>15000 / \mathrm{mm}^{3} *$ & 0.7170 & 2.0 & $1.0-4.2$ & .0515 & 1 \\
\hline Liver cirrhosis & -1.2336 & 0.2 & $0.1-1.1$ & .0671 & -2 \\
\hline
\end{tabular}

* Nonpredictors or different assigned scores in comparing to CZ-RES model.

16.9 years (mean $\pm \mathrm{SD}$ ). In the univariate analysis, men were more likely to develop CZ-RES (odds Ratio [OR], $1.5 ; 95 \% \mathrm{CI}, 1.1-2.3$ ) compared to women, but this was not statistically significant in the multivariate regression. Some underlying comorbidities also showed a strong association with resistance, in particular, having a history of stroke with repeated choking or hematological malignancy. Cirrhosis of liver showed a significant inverse association with CZ-RES but not with CTX-RES in univariate analysis. Many of the hospital exposure and health care-related factors were significantly associated with elevated risk of developing resistance, the strongest indicators being time since last hospitalization and previous infection with a resistant strain. Of the initial presentations, we found that abnormal vital signs, and abnormally high or low complete blood cell and differential counts were associated with greater risk. Poor oxygen saturation was related to CTXRES but not to CZ-RES.

Final predictors and assigned coefficient-based scores for CZ-RES model and CTX-RES model were listed in Table 2 and Table 3. The covariates for CZ-RES model were discharge from hospital within the last month, prior

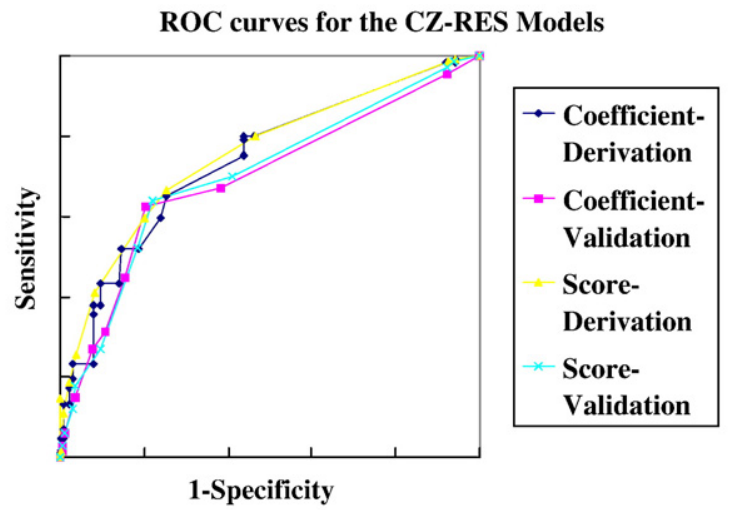

The approximation of four curves indicates the proximate predictive performance of coefficient-based and score-based models, both in derivation and validation dataset.

Fig. 3 The ROC curves for assessing predictive validity of CZRES models. infection with a CTX-RES strain, residence in a nursing home or history of stroke with repeated choking, use of immunosuppressants in transplantation patients, leucopenia $[<1000(1 / \mathrm{mm})]$ or leukocytosis $[>15000(1 / \mathrm{mm})]$ at first blood test, and poor oxygen saturation $\left(\mathrm{SpO}_{2}<95 \%\right)$. Surprisingly, after adjusting for other factors in the multivariate analysis, cirrhosis of the liver had a protective effect with regard to CZ-RES and was retained in the final model. For ease of clinical use, it was also kept in the final CTX-RES model, although it was only marginally significant there. Predictors included in the final CTX-RES model were all those already included in the CZ-RES model plus abnormal leukocyte count $(<1000$ or $>15000$ / $\mathrm{mm}^{3}$ ) at first blood test. Besides, shorter interval since prior hospitalization, particularly within 2 weeks, led to an increase risk for antimicrobial-resistant bacteremia. Hosmer-Lemeshow goodness-of-fit test shows a satisfactory model fitting $(P=.83$ for CZ-RES and $P=.28$ for CTX-RES).

The ROC curves for the CZ-RES and CTX-RES models were plotted in Fig. 3 and Fig. 4, respectively. For the CZRES models, the AUC of the primary coefficient model

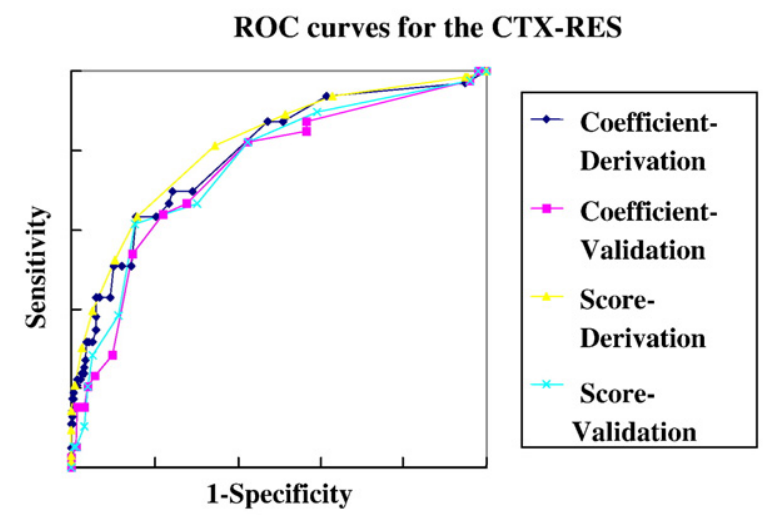

The approximation of four curves indicates the proximate predictive performance of coefficient-based and score-based models, both in derivation and validation dataset.

Fig. 4 The ROC curves for assessing predictive validity of CTXRES models. 
Table 4 CZ-RES scoring model: predictive performance for different scores

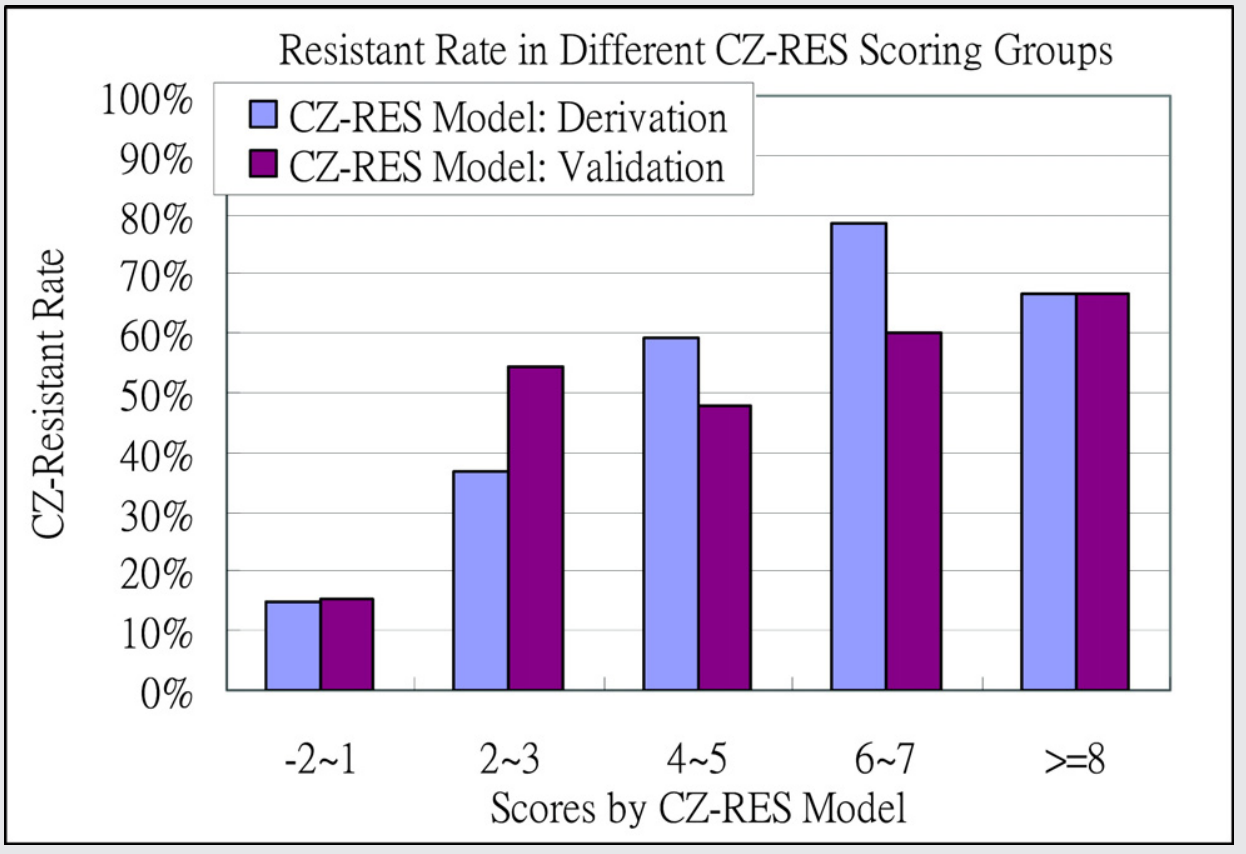

\begin{tabular}{|c|c|c|c|c|c|c|c|}
\hline \multicolumn{2}{|c|}{ Range of scores } & \multirow{2}{*}{$\frac{-2 \text { to } 1}{43}$} & \multirow{2}{*}{$\frac{2-3}{33}$} & \multirow{2}{*}{$\frac{4-5}{29}$} & \multirow{2}{*}{$\frac{6-7}{22}$} & \multirow{2}{*}{$\frac{\geq 8}{2}$} & \multirow{2}{*}{$\frac{\text { Total }}{129}$} \\
\hline Derivation & CZ-RES & & & & & & \\
\hline & Subtotal & 290 & 90 & 49 & 28 & 3 & 460 \\
\hline & Risk & $15 \%$ & $37 \%$ & $59 \%$ & $79 \%$ & $67 \%$ & $28 \%$ \\
\hline \multirow[t]{3}{*}{ Validation } & CZ-RES & 24 & 25 & 10 & 6 & 2 & 67 \\
\hline & Subtotal & 155 & 46 & 21 & 10 & 3 & 235 \\
\hline & Risk & $15 \%$ & $54 \%$ & $48 \%$ & $60 \%$ & $67 \%$ & $29 \%$ \\
\hline
\end{tabular}

(abbreviating to coefficient derivation) was 0.76 (95\% CI, 0.71-0.81). For the CTX-RES models, the AUC of the primary coefficient model (coefficient derivation) was 0.82 (95\% CI, 0.76-0.88). The closeness of the 4 ROC curves (coefficient and score models applied to both the derivation and validation data sets) in both models suggests good predictive validity.

To develop the scoring models into a practical tool for clinical decision making, the optimal cut points were identified, and the predictive accuracy at these points was assessed. For the CZ-RES model, from the derivation data, we estimated this to be "score $\geq 2$ " (giving sensitivity and specificity of $67 \%$ and $75 \%$, respectively), and from the validation set, "score $\geq 3$ " (reducing sensitivity to $60 \%$ but increasing specificity to $80 \%$ ). Applying the same approach to the CTX-RES model, we found the equivalent optimal cut points to be score $\geq 3$ (yielding $81 \%$ sensitivity and $65 \%$ specificity) and "score $\geq 4$," which yielded $63 \%$ sensitivity and $84 \%$ specificity.

To assess whether the performance of this decision tool is adequate for clinical use, we ascertained its predictive ability for a range of different scores. As shown in Tables 4 and 5 , the overall proportion in the derivation and validation sets with CZ-RES GNB was 28\% and 29\%, respectively. However, the results by score indicate that the proportion with resistant infection increases with score and that the decision rules, therefore, has good predictive properties. There was a slight decrease in the proportion with resistant infection in the "score $\geq 8$ " group, but this may well be due to the fact that so few were in this category. Similarly, the proportion with CTX-RES GNB in the derivation and validation data sets was $14 \%$ and $17 \%$ respectively. Again, the score $\geq 8$ group was relatively small, but the resistant rate otherwise increased with score. Thus, it appears that the decision rules we developed do have sufficient predictive power to make them useful in clinical setting, that is, they are able to differentiate between the cases most likely to have resistant or nonresistant disease.

\section{Discussion}

In this study, we used a derivation-validation method to develop 2 decisions rules to predict antibiotic-resistant GNB in patients at ED by a simple scoring system that uses only the clinical information that is readily available 
Table 5 CTX-RES scoring model: predictive performance for different scores

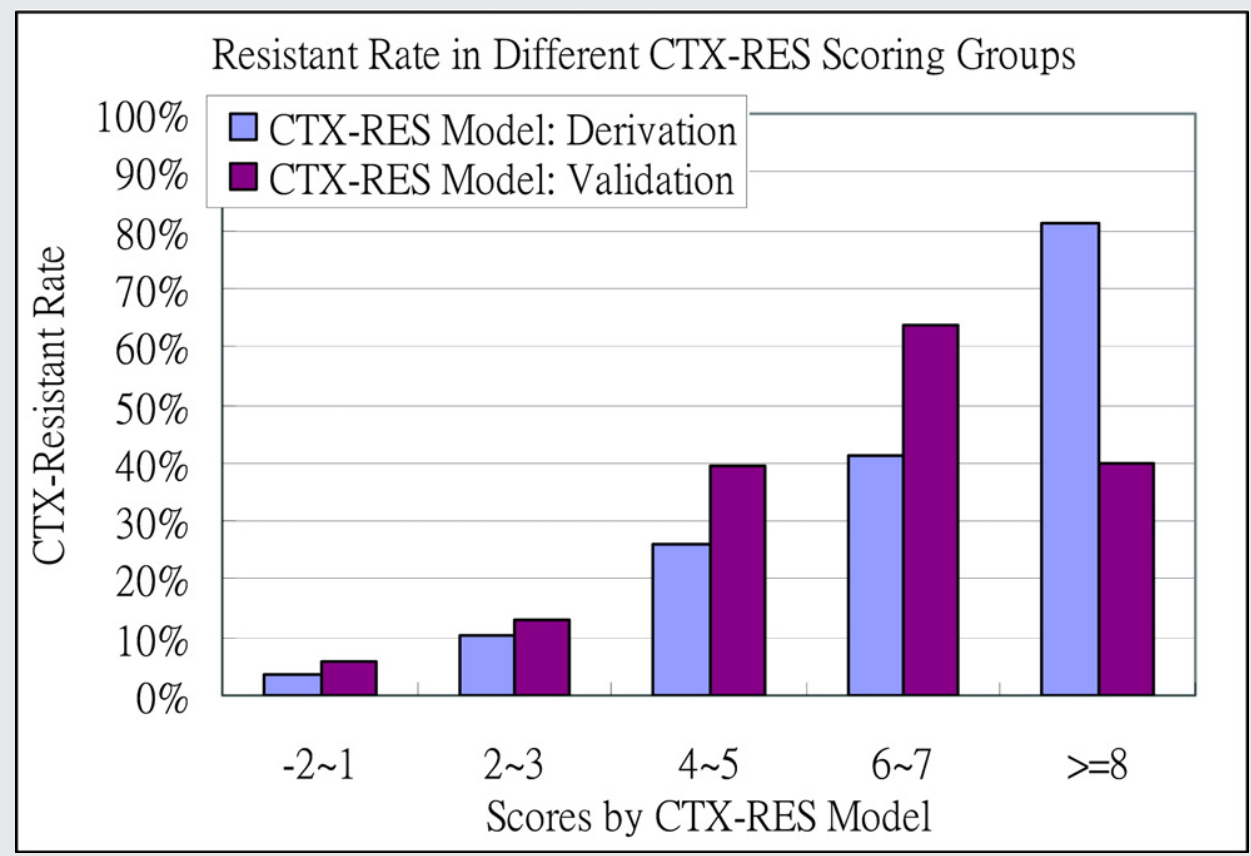

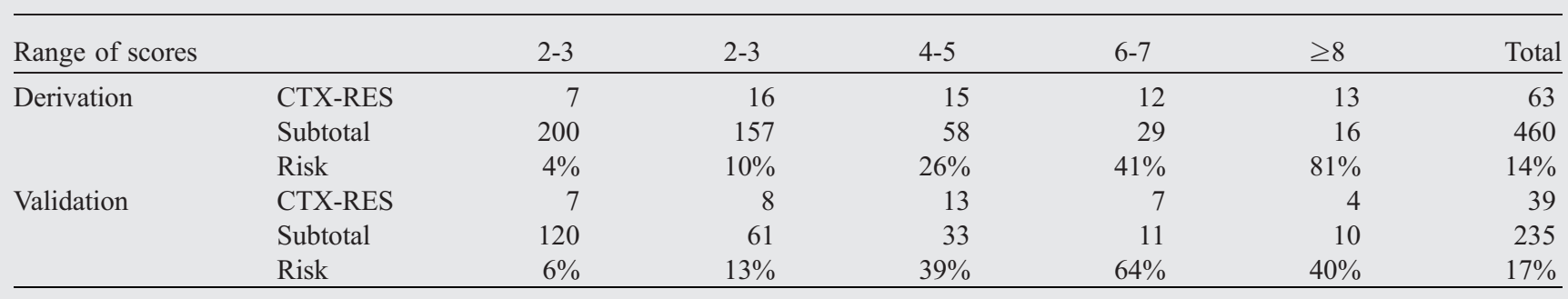

at the time. We tried to adhere to the standard criteria in development of prediction model [26,27]. To the best of our knowledge, it is also the first study to elucidate the association between clinical risk factors and antimicrobial resistance in Taiwan. Our prediction models appear to be useful for clinical practice by incorporating relevant risk factors.

Because GNB may ensue from inadequate antibiotic treatment, the choice of first-, second-, or third-generation antibiotic is of paramount importance, yet the decision often has to be made before the culture result is available. Physicians tend to prescribe a second generation of antibiotic for infected patients with risk factors suggestive of resistance. However, if the patient is stable clinically and the doctor is unaware of a risk factor (eg, recent hospitalization within the last month), he may be in a dilemma as to which antibiotic to prescribe. Our CZ-RES and CTX-RES models, which were derived from a large study according to respected scientific guidelines, provide a good evidence-based reference in this circumstance. Such a model would enable physicians to commence treatment earlier and therefore reduce morbidity, mortality, and the related economic costs.
Hospital exposure and health care-associated factors, as widely documented in previous literature, were also significant in our cohort. Friedman et al [11] found that patients living in nursing home had similar bloodstream infections to those in hospital settings, particularly methicillin-resistant Staphylococcus aureus. However, the antibiotic resistance of Gram-negative pathogens was not further analyzed in their study. From a case-control study, Schippa et al [13] reported an OR of 3.6 for ceftazidimeresistant GNB in nursing home residents, comparing to general population. In our prospective cohort, although nursing home residence was significant in univariate analysis, it lost significance after being adjusted by other covariates (OR, 1.6; 95\% CI, 0.6-6.2). Similar results were found for history of stroke with repeated choking, but after dealing with the problem of collinearity of these 2 variables by selecting a composite measure, the factor turned out to be significant in final models.

Associations between initial clinical presentation and antimicrobial resistance have barely been addressed before. Leukocytopenia $\left(<1000 / \mathrm{mm}^{3}\right)$ has long been suspected of being related to serious Gram-negative infection (such as Pseudomonas spp). Besides, Jaimes et al [30] found 
leukocytosis $\left(>12000 / \mathrm{mm}^{3}\right)$ to be an independent predictor of positive bacteremia but did not investigate the effect on antibiotic resistance. We found that leucopenia $(<1000 /$ $\left.\mathrm{mm}^{3}\right)$ and leukocytosis $\left(>15000 / \mathrm{mm}^{3}\right)$ were both associated with antimicrobial-resistant GNB. Theoretically, bacteremia leads to a more severe systemic response than focal infections, but whether cephalosporin resistant pathogens have the same effect on each case is still under investigation. Poor oxygen saturation was also related to antimicrobial resistance in our study, probably because of its association with severe pneumonia. However, because the infectious focus might be unknown initially at ED, we did not take this variable (ie, final diagnosis) into account when developing our prediction rules.

Liver cirrhosis was inversely associated with cephalosporin-resistant GNB in our study, although many studies have emphasized that their outcomes are poorer in comparison to the general population. The poor outcome is perhaps due to the severity of disease rather than the infectious pathogen [31-34]. Graudal et al reported that there was no difference of bacterial strains when bacteremia occurred in cirrhotic patients versus noncirrhotic patients [35]. Because of chronic hepatitis B viral infection, liver cirrhosis is relatively common in the Chinese and Taiwanese. Our study includes a larger proportion of cirrhotic patients than would be found in western studies, which may partly explain the novel finding of inverse association. Of course, the biologic rationale should be clarified in future research.

There are several limitations to this study. Firstly, there is a lack of clinical data from patients with negative blood cultures at ED. Thus, the decision rules developed here are not applicable to all ED patients with suspected blood infection because the ED deals with more than 100000 patients per year and our research team was unable to follow-up those whose blood cultures turned out to be negative. Secondly, we did not collect information on the antimicrobial agents prescribed by the primary care physicians at ED. If this data were available, we would have been able to compare the accuracy of our model predictions with that of clinician judgment. Thirdly, the questionnaire used to collect medical history data was constructed by the principal researchers specifically for this study, so it was never formally validated. Finally, because our samples were derived from the ED of a tertiary hospital, our results may not be representative of patients in other community hospitals. Antimicrobial resistance is an extremely complex problem worldwide. Multicenter studies may be needed in the future.

\section{Conclusion}

We have developed 2 models for predicting risk of antimicrobial Gram-negative infection by identifying and quantifying associated risk factors. These models could be used by physicians to determine the most appropriate choice of antibiotic for first-line therapy, particularly in situations where the culture result is not yet known.

\section{References}

[1] Carmeli Y, Troillet N, Karchmer AW, et al. Health and economic outcomes of antibiotic resistance in Pseudomonas aeruginosa. Arch Intern Med 1999;159:1127-32.

[2] Lautenbach E, Patel JB, Bilker WB, et al. Extended-spectrum betalactamase-producing Escherichia coli and Klebsiella pneumoniae: risk factors for infection and impact of resistance on outcome. Clin Infect Dis 2001;32:1162-71.

[3] Virk A, Steckelberg JM. Clinical aspects of antimicrobial resistance. Mayo Clin Proc 2000;75:200-14.

[4] Zuccotti GV, Gracchi V, Sala D, et al. Nosocomial infections at a pediatric age. Pediatr Med Chir 2002;24:177-85.

[5] Weinstein MP, Towns ML, Quartey SM, et al. The clinical significance of positive blood cultures in the 1990s: a prospective comprehensive evaluation of the microbiology, epidemiology, and outcome of bacteremia and fungemia in adults. Clin Infect Dis 1997;21:584-602.

[6] Pop-Vicas AE, D'Agata EM. The rising influx of multidrug-resistant Gram-negative bacilli into a tertiary care hospital. Clin Infect Dis 2005;40:1792-8

[7] Lucet JC, Chevert S, Decre D, et al. Outbreak of multiply resistant Enterobacteriaceae in an intensive care unit: epidemiology and risk factors for acquisition. Clin Infect Dis 1996;22:430-6.

[8] De Champs C, Rouby D, Guelon D, et al. A case-control study of an outbreak of infections caused by Klebsiella pneumoniae strains producing CTX-1(TEM-3) beta-lactamase. J Hosp Infect 1991; 18:5 - 13 .

[9] Paterson DL, Mulazimoglu L, Casellas JM, et al. Epidemiology of ciprofloxacin resistance and its relationship to extended-spectrum beta-lactamase production in Klebsiella pneumoniae isolates causing bacteremia. Clin Infect Dis 2000;30:437-8.

[10] Wiener J, Quinn JP, Bradford PA, et al. Multiple antibiotic-resistant Klebsiella pneumoniae and Escherichia coli in nursing home. JAMA 1999;281:517-23.

[11] Friedman ND, Kaye KS, Stout JE, et al. Health care-associated bloodstream infections in adults: a reason to change the accepted definition of community-acquired infections. Ann Intern Med 2002;137:791 - 7

[12] Siegman-Igra Y, Fourer B, Orni-Wasserlauf R, et al. Reappraisal of community-acquired bacteremia: a proposal of a new classification for the spectrum of acquisition of bacteremia. Clin Infect Dis 2002; 34:1431-9.

[13] Schiappa DA, Hayden MK, Matushek MG, et al. Ceftazidimeresistant Klebsiella pneumoniae and Escherichia coli bloodstream infection: a case-control and molecular epidemiologic investigation. J Infect Dis 1996;174:529-36.

[14] Blot S, Vandewoude K, De Bacquer D, et al. Nosocomial bacteremia caused by antibiotic-resistant Gram-negative bacteria in critically ill patients: clinical outcome and length of hospitalization. Clin Infect Dis 2002;34:1600-6.

[15] Pena C, Pujol M, Ricart A, et al. Risk factors for faecal carriage of Klebsiella pneumoniae producing extended spectrum beta-lactamase (ESBL-KP) in intensive care unit. J Hosp Infect 1997;35:9-16.

[16] Piroth L, Aube H, Doise JM, et al. Spread of extended-spectrum beta-lactamase-producing Klebsiella pneumoniae: are betalactamase inhibitors of therapeutic value? Clin Infect Dis 1998; 27:76-80.

[17] Ho M, Hsiung CA, Yu HT, et al. Antimicrobial usage in ambulatory patients with respiratory infections in Taiwan, 2001. J Formos Med Assoc 2004;103:96-103. 
[18] Goldberg D. The cephalosporins. Med Clin North Am 1987; 71:1113.

[19] Gantz NM, Brown R, Berk SL, Esposito AL, et al. Manual of clinical problems in infectious disease. Chap. 74: choosing a cephalosporin, 4th ed. 1999. p. $410-4$.

[20] MacDonald KL, Cohen ML, Hargrett-Bean NT, et al. Changes in antimicrobial resistance of Salmonella isolated from humans in the United States. JAMA 1987;11:1496-9.

[21] Ackers ML, Puhr ND, Tauxe RV, et al. Laboratory-based surveillance of Salmonella serotype Typhi infections in the United States: antimicrobial resistance on the rise. JAMA 2000;283:2668-73.

[22] Ko WC, Lee HC, Chuang YC, et al. Clinical features and therapeutic implications of 104 episodes of monomicrobial Aeromonas bacteraemia. J Infect 2000;40:267-73.

[23] Weber JT, Mintz ED, Canizares R, et al. Epidemic cholera in Ecuador: multidrug-resistance and transmission by water and seafood. Epidemiol Infect 1994;112:1 - 11 .

[24] van den Bogaard AE, Stobberingh EE. Antibiotic usage in animals: impact on bacterial resistance and public health. Drugs 1999;58:589-607.

[25] Sharma H, Unicomb L, Forbes W, et al. Antibiotic resistance in Campylobacter jejuni isolated from humans in the Hunter Region, New South Wales. Commun Dis Intell 2003;27(Suppl):S80-8.

[26] Hanley JA, McNeil BJ. The meaning and use of the area under a receiver operating characteristic (ROC) curve. Radiology 1982;143:29-36.
[27] Wasson JH, Sox HC, Neff RK, et al. Clinical prediction rules. Applications and methodological standards. N Engl J Med 1985;313:793-9.

[28] Laupacis A, Sekar N, Stiell IG. Clinical prediction rules. A review and suggested modifications of methodological standards. JAMA 1997;277:488-94

[29] Sullivan LM, Massaro JM, D'Agostino Sr RB. Presentation of multivariate data for clinical use: The Framingham Study risk score functions. Stat Med 2004;23:1631-60.

[30] Jaimes F, Arongo C, Ruiz G, et al. Predicting bacteremia at the bedside. Clin Infect Dis 2004;38:357-62.

[31] Aloy Duch A, Espejo Arenas E, Mauri Pont M, et al. Bacteremia in the patient with liver cirrhosis. Prospective study of 61 episodes. Enferm Infecc Microbiol Clin 1990;8:540 - 3.

[32] Kuo CH, Changchien CS, Yang CY, et al. Bacteremia in patients with cirrhosis of the liver. Liver 1991;11:334-9.

[33] Mizuno R, Nishiyama Y, Shimizu S, et al. Clinical study on bacteremia in patients with liver cirrhosis. Kansenshogaku Zasshi 1996;70:456-62.

[34] Thulstrup AM, Sorensen HT, Schonheyder HC, et al. Population-based study of the risk and short-term prognosis for bacteremia in patients with liver cirrhosis. Clin Infect Dis 2000;31:1357-61.

[35] Graudal N, Milman N, Kirkegaard E, et al. Bacteremia in cirrhosis of the liver. Liver 1986;6:297-301. 